\title{
Factors associated with the development of screw cut-out after the fixation of intertrochanteric femoral fractures with a proximal femoral nail
}

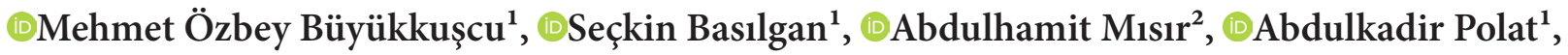 \\ (D) Hakan Başar ${ }^{1}$ \\ ${ }^{1}$ Health Science University, İstanbul Gaziosmanpasa Training and Research Hospital, Department of Orthopaedics and Traumatology, \\ İstanbul, Turkey \\ ${ }^{2}$ Health Science University, İstanbul Başakșehir Pine and Sakura City Training and Research Hospital, Department of Orthopaedics and \\ Traumatology, İstanbul, Turkey
}

Cite this article as: Büyükkuşcu MÖ, Basılgan S, Mısır A, Polat A, Başar H. Factors associated with the development of screw cut-out after the fixation of intertrochanteric femoral fractures with a proximal femoral nail. J Health Sci Med 2021; 4(2): 170-175.

\begin{abstract}
Objective: To reveal the factors associated with screw cut-out in the fixation of proximal femur intertrochanteric fractures with a proximal femoral nail (PFN).

Material and Method: Patients who were diagnosed with proximal femoral intertrochanteric fractures and were being treated and followed up in our hospital between January 2014 and January 2019 were retrospectively analyzed. The hip fracture types of the patients were determined according to the American Foundation/American Orthopedic Trauma Association (AO/OTA) classification. AO/OTA 31-A1, A2 and A3 type fractures were included in the study. Twenty-seven patients with PFN fixation failure and screw cut-out (cut-out group, 11.4\%) were compared with 208 patients who had successful osteosynthesis without cut-out (non-cut-out group, 88.6\%). Age, gender, affected side, follow-up time, PFN design, tip-apex distance (TAD), calcar-referenced TAD, fracture type, reduction quality, posteromedial support loss, lag screw position, Singh index, and collo-diaphyseal angle (CDA) were compared between the two groups.

Results: The two groups significantly differed in terms of TAD and calcar-referenced TAD ( $p=0.002$ and 0.001 , respectively). In the evaluation of reduction quality according to the Baumgaertner scale and the Garden alignment index, a significant difference was found between the two groups ( $\mathrm{p}=0.021$ and 0.002 , respectively). A significant difference was also observed between the two groups in terms of screw position and posteromedial cortex continuity ( $\mathrm{p}=0.009$ and 0.037 , respectively). However, there was no significant difference in relation to age, gender, affected side, CDA, PFN design, and osteoporosis severity.

Conclusion: Fracture type, poor reduction quality, loss of posteromedial support, TAD, calcar-referenced TAD, and lag screw position were found to be associated factors in the development of screw cut-out. Apart from the type of fracture, these factors that are under the control of the surgeon generally show the importance of anatomical reduction and accurate screw placement. According to the results obtained, the risk of screw cut-out can be reduced by preoperative planning and intraoperative evaluation.
\end{abstract}

Keywords: Intertrochanteric femoral fractures, proximal femoral nail, associated factors, implant failure, cut-out

\section{INTRODUCTION}

Hip fractures are a major cause of increased mortality and morbidity, especially in the elderly. Trochanteric hip fractures constitute about half of all hip fractures (1). According to the American Foundation/American Orthopedic Trauma Association (AO/OTA) classification system, fractures involving the trochanteric region of the proximal femur are classified as AO/OTA 31-A. These fractures are further divided into the groups of A1, A2 and A3. A1 is defined as simple, two-part fractures; A2 fractures are those with multiple fragments; and A3 refers to more complex fractures, including reverse oblique and transverse fracture patterns (2).
Current treatment options for intertrochanteric fractures are cephalomedullary nails and dynamic hip screws, with the former being considered to be superior to the latter (3). Cephalomedullary nails are the preferred implants, especially since they allow for acceptable closed reduction. In addition, intramedullary fixation is associated with reduced soft tissue trauma, decreased blood loss, and lower infection rates and wound complications (4). On the other hand, the use of the proximal femoral nail (PFN) is associated with certain complications, including thigh pain, displacement of interlocking head screws (Z-effect and reverse Z-effect), varus collapse, screw cut-out, peri- 
implant fracture, non-union, delayed union, femoral neck shortening, and infection development. The most frequently reported complication in PFN application in hip fractures is screw cut-out, which is defined as the extrusion of the screw from the femoral head with the varus collapse of the femoral neck (5).

The aim of this study was to discuss the factors associated with screw cut-out, which is the most common complication in fixation with $\mathrm{PFN}$, which remains controversial in the literature. We hypothesized that the development of screw cut-out was related to factors under the surgeon's control and therefore they could be altered.

\section{MATERIAL AND METHOD}

The study was approved by Gaziosmanpaşa Training and Research Hospital Clinical Researchs Ethics Committee (approval date and number: 02.12.2020/192). The study was conducted in accordance with the principles of the Declaration of Helsinki. Patients who were diagnosed with proximal femoral intertrochanteric fractures and were being treated and followed up in our hospital between January 2014 and January 2019 were retrospectively analyzed. Patients under 65 years of age $(n=42)$, those with pathological fractures ( $n=13$ patients) or ipsilateral knee or ankle fractures $(n=2)$, those that had undergone open reduction $(n=24)$, those without a follow-up for at least one year $(n=51)$, and cases in which a fixation material other than PFN was used $(n=21)$ were excluded from the study. The hip fracture types of the patients included in the study were determined according to the AO/OTA classification system (4), and only AO/OTA 31- A1, A2 and A3 type fractures were included in the sample. Trigen InterTan (Smith \& Nephew, Memphis, TN, USA) nails were used in 61 patients and Profin (TST SAN, İstanbul, Turkey) nails in 26 patients.
Twenty-seven patients with PFN fixation failure and screw cut-out were evaluated as the cut-out group and 208 patients with successful osteosynthesis constituted the non-cut-out group. Age, gender, affected side, tip-apex distance (TAD), fracture type, reduction quality, loss of posteromedial support, lag screw position, angle between the lag screw and femoral neck axis, Singh index, neck-shaft angle, and the Garden alignment index (GAI) were compared.

Preoperative and postoperative anteroposterior (AP) and lateral radiographs were used to classify the fractures and determine the quality of fracture reduction, collodiaphyseal angle (CDA), lag screw position, TAD (5), and calcar-referenced TAD (CalTAD) (6). Fracture reduction was evaluated in the first postoperative radiograph using GAI (7). GAI was used to define the angle between neck and shaft in AP and lateral view. The results were classified as very good (AP $\left.160^{\circ}\right)$, good (AP 180-160 $)$, acceptable (AP $\left.160-150^{\circ}\right)$ and poor $\left(\mathrm{AP}<150^{\circ}\right)$. The postoperative reduction quality of the patients was determined as good, acceptable, or poor according to the reduction criteria defined by Baumgaertner et al. (5). The state of posteromedial support was defined as present or absent according to the amount of the displacement of the posteromedial segment. A displacement of less than the cortical thickness means that there is contact between proximal and distal fragments, and it is interpreted as the presence of posteromedial support (8).

The osteoporosis severity of the patients was evaluated according to the Singh index (9). The lag screw position was determined according to the method described by Cleveland (Figure 1) (10). Radiographic measurements were performed at two separate times in a month by two different orthopedic surgeons. High intra-observer [intraclass correlation coefficient (ICC) 0.91 (95\% CI 0.82-0.96)] and inter-observer (ICC 0.83 [95\% CI 0.810.93 ] agreement was observed in the measurements.

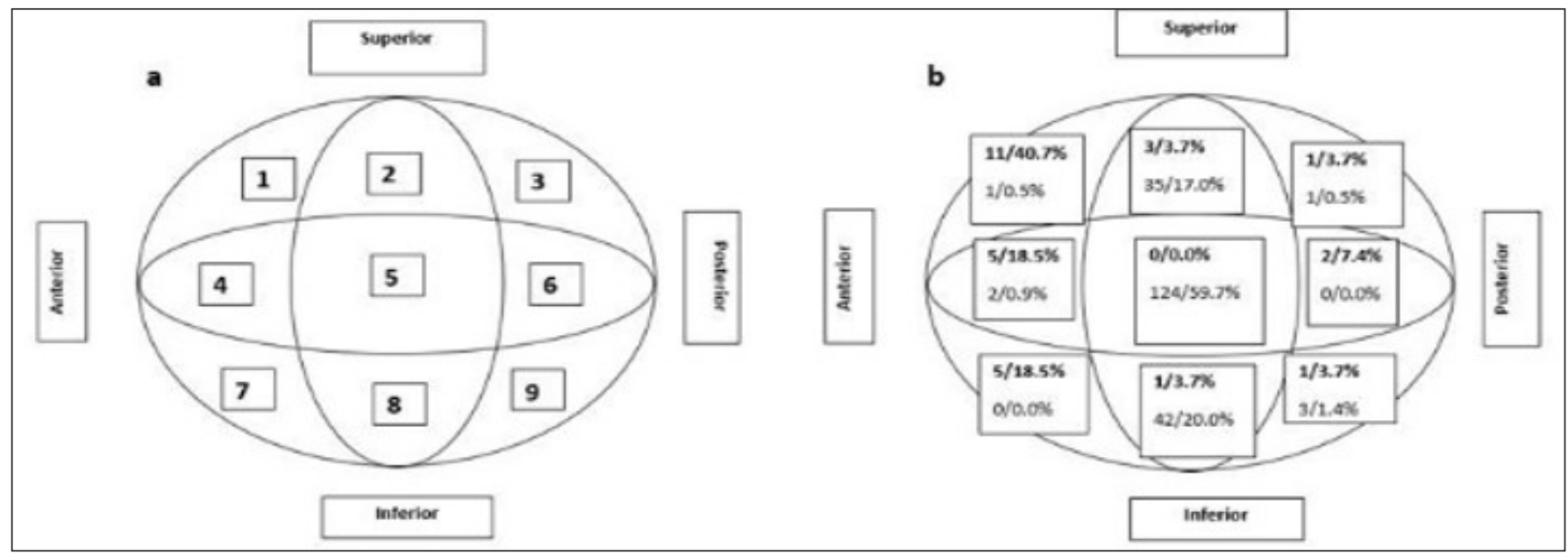

Figure 1. Lag screw positioning within the femoral head. a: Cleveland's definition of the location of lag screw in the femoral head. The ninezone template of the head, b: The number of implant failures and failure rate in each zone is represented. Bold-italics indicates the cut-out group values. 


\section{Surgical Protocol}

All the patients underwent surgery under general or regional anesthesia on the traction table in the supine position. Closed reduction and minimally invasive nailing were performed under fluoroscopic imaging. The patients then underwent routine surgical procedures for PFN implantation according to the manufacturer's protocol. Both PFN types had trochanter major tips. Postoperatively, the patients received standard prophylaxis for deep vein thrombosis. Partial weight bearing was initiated after fracture healing was seen on radiographs, and total weight bearing was allowed once clinical fracture healing was observed. Follow-up evaluations were undertaken at postoperative months 1, 3, 6 and 12, and annually thereafter.

\section{Statistical Analysis}

Statistical analyses were performed using SPSS v. 22.0 (SPSS Inc., IBM, NY, USA). The distribution of variables was analyzed using the Kolmogorov-Smirnov test. The independent-samples t-test was used for the comparison of continuous quantitative data with a normal distribution, the Mann-Whitney $U$ test was used to compare continuous quantitative data without a normal distribution, and the chi-square or Fischer's exact test was conducted for the comparison of categorical data. The level of significance was set at $\mathrm{p}<0.05$ for all statistical tests. A post-hoc power analysis was performed to determine whether the number of patients in each group was adequate to achieve statistical significance and to avoid type II error. More than $80 \%$ power was detected for the comparison of the measured parameters between the groups. ICC with a 95\% confidence interval was used to quantify intra- and inter-observer agreement regarding the measured parameters. Based on the study of Landis and Koch (11), we defined an ICC value of $0-0.2$ as indicating slight agreement, 0.21-0.40 fair agreement, 0.410.60 moderate agreement, $0.61-0.8$ substantial agreement, and $>0.80$ perfect agreement.

\section{RESULTS}

Between January 2014 and January 2019, 386 consecutive trochanteric fractures patients underwent surgery in our institution. The mean age of the patients was $78.91 \pm 10.71$ (range, 65-95) years, and the mean follow-up time was $30.1 \pm 6.9$ (12-70) months. Screw cut-out was seen in 27 (11.4\%) of 235 patients. Four (14.8\%) patients underwent plate-screw fixation, twelve (44.2\%) patients underwent partial hip replacement and $11(41 \%)$ required revision surgery with total hip replacement (Figure 2). The demographic characteristics and clinical data of the patients are summarized in Table 1. There was no significant difference between the two groups in terms of age, gender, and affected side (Table 1). There was also no significant difference between the two groups in relation to CDA, PFN design, and osteoporosis severity $(\mathrm{p}=0.156, \mathrm{p}=0.218$, and $\mathrm{p}=0.291$, respectively) (Table 2 and Table 3 ).

\begin{tabular}{|c|c|c|c|}
\hline & $\begin{array}{l}\text { Cut-out Group } \\
\quad(n=27)\end{array}$ & $\begin{array}{l}\text { Non-cut-out Group } \\
(n=208)\end{array}$ & $\mathbf{p}$ \\
\hline Age & $76.85 \pm 9.11(65-91)$ & $80.08 \pm 11.21(65-95)$ & 0.475 \\
\hline \multicolumn{4}{|l|}{ Affected side } \\
\hline Right & $16(59.2 \%)$ & $102(48.3 \%)$ & 0.487 \\
\hline Left & $11(40.8 \%)$ & $106(51.7 \%)$ & 0.262 \\
\hline \multicolumn{4}{|l|}{ Gender } \\
\hline Male & $10(37.0 \%)$ & $87(41.6 \%)$ & 0.814 \\
\hline Female & $17(63.0 \%)$ & $121(58.4 \%)$ & 0.434 \\
\hline $\begin{array}{l}\text { Follow-up } \\
\text { time (months) }\end{array}$ & $28.1 \pm 5.6(12-66)$ & $31.3 \pm 7.7(13-70)$ & 0.706 \\
\hline $\begin{array}{l}\text { AO/OTA } \\
\text { classification }\end{array}$ & & & 0.011 \\
\hline 31-A1 & $2(7.4 \%)$ & $142(68.2 \%)$ & \\
\hline 31-A2 & $18(66.6 \%)$ & $34(16.3 \%)$ & \\
\hline 31-A3 & $7(26.0 \%)$ & $32(14.5 \%)$ & \\
\hline
\end{tabular}

\begin{tabular}{|c|c|c|c|}
\hline & $\begin{array}{c}\text { Cut-out Group } \\
(\mathbf{n}=27)\end{array}$ & $\begin{array}{l}\text { Non-cut-out Group } \\
\qquad(\mathrm{n}=208)\end{array}$ & $\mathbf{p}$ \\
\hline Mean TAD (mm) & $33.19 \pm 7.64$ & $22.18 \pm 4.58$ & 0.002 \\
\hline Mean CaIDAT $(\mathrm{mm})$ & $29.74 \pm 7.33$ & $23.32 \pm 7.33$ & 0.001 \\
\hline Mean CDA $\left(^{\circ}\right)$ & $132.67 \pm 10.79$ & $135.82 \pm 8.56$ & 0.156 \\
\hline Singh index & $3.37 \pm 0.92$ & $3.63 \pm 0.75$ & 0.291 \\
\hline \multicolumn{4}{|c|}{$\begin{array}{l}\text { Bold values indicate statistical significance. } \mathrm{P}<0.05 \\
\text { (TAD: Tip-apex distance, CDA: Collo-diaphyseal angle, CaIDAT: Calcarreferenced tip- } \\
\text { apex distance) }\end{array}$} \\
\hline
\end{tabular}

Table 3. Comparison of the groups in terms of nail design and reduction quality

\begin{tabular}{|lccc|}
\hline & $\begin{array}{c}\text { Cut-out } \\
\text { Group } \\
(\mathbf{n = 2 7})\end{array}$ & $\begin{array}{c}\text { Non-cut- } \\
\text { out Group } \\
(\mathbf{n = 2 0 8})\end{array}$ & $\mathbf{p}$ \\
\hline $\begin{array}{l}\text { Proximal Femoral Nail Design } \\
\text { InterTan (intertrochanteric } \\
\text { antegrade nail) }\end{array}$ & $16(59.2 \%)$ & $145(70.0 \%)$ & 0.218 \\
$\begin{array}{l}\text { Profin (proximal femoral } \\
\text { nail) }\end{array}$ & $11(40.8 \%)$ & $63(30.0 \%)$ & \\
$\begin{array}{l}\text { Posteromedial cortex continuity } \\
\text { Existence }\end{array}$ & $8(29.7 \%)$ & $116(56.7 \%)$ & \\
Loss & $19(70.3 \%)$ & $92(43.3 \%)$ & \\
Garden alignment index & $3(11.1 \%)$ & $131(62.9 \%)$ & \\
Very good & $6(22.2 \%)$ & $57(27.4 \%)$ & \\
Good & $7(26.7 \%)$ & $16(7.8 \%)$ & \\
Acceptable & $11(40.0 \%)$ & $4(1.9 \%)$ & \\
Poor & & & 0.037 \\
Baumgaertner Reduction Quality & $5(18.5 \%)$ & $176(84.6 \%)$ & \\
Good & $8(29.6 \%)$ & $29(13.9 \%)$ & \\
Acceptable & $14(51.9 \%)$ & $3(1.5 \%)$ & \\
Poor & & & \\
\hline P $<0.05$ & & & \\
\hline
\end{tabular}




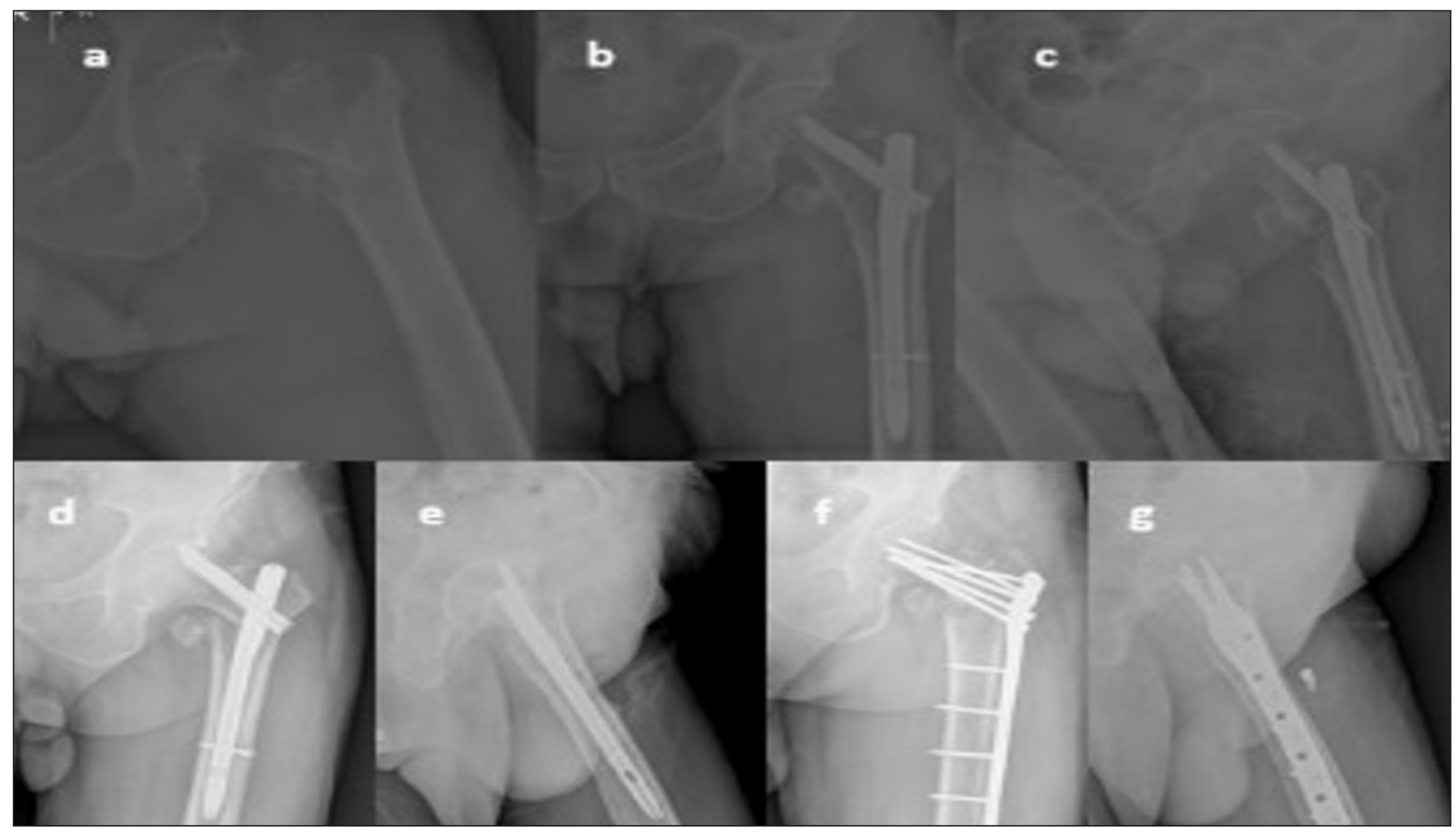

Figure 2. 68-year-old male patient who has AO type 31-A2 femur fracture. a: a preoperative anteroposterior hip radiography, b-c: early postoperative anteroposterior and lateral hip radiographs show fracture reduction and fxation using PFN, d-e: postoperative anteroposterior and lateral hip radiographs show screw cut-out, f-g: Image of loss of reduction in the patient's first month follow-up radiography. Revision surgery with plate-screw was applied as a salvage option.

The groups significantly differed in respect of fracture types according to the AO/OTA classification ( $\mathrm{p}=0.011$ ) (Table 1). There were also significant differences in terms of TAD and CaITAD values $(\mathrm{p}=0.002$ and 0.001 , respectively) (Table 2 ). When reduction quality was evaluated according to the Baumgaertner scale, a significant difference was observed between the two groups ( $\mathrm{p}=0.021$ ). Similarly, according to GAI, there was a significant difference in the reduction quality of the two groups $(\mathrm{p}=0.002)$ (Table 3). A further significant difference was found in relation to posteromedial cortex continuity ( $\mathrm{p}=0.037)$ (Table 3 ).

According to the locations defined by Cleveland, the lag screw position was zone 1 in $11(40.7 \%)$ patients, zone 4 in five (18.5\%) patients, and zone 7 in five (18.5\%) patients in the cut-out group. In the non-cut-out group, the lag screw position was zone 2 for 35 (17\%) patients, zone 5 for 124 (59.7\%), and zone 8 for 42 (20\%). There was a significant difference between the two groups in terms of lag screw position $(\mathrm{p}=0.009)$ (Figure 2).

\section{DISCUSSION}

The most important finding of this study was the association of postoperative cut-out development with fracture type, reduction quality, TAD, CaITAD, loss of posteromedial support, and lag screw position in the fixation of intertrochanteric hip fractures with
PFN. In addition, the screw cut-out complication rate was determined as $11.4 \%$ in PFN applications among the 235 consecutive patients with proximal femoral intertrochanteric fractures.

\section{TAD and CaITAD}

The literature reports that there is a correlation between implant cut-out and TAD and CaITAD in hip fractures. Baumgaertner et al. (5) determined that the TAD value was the best predictive parameter in determining the cutout rates during a three-month follow-up period $(6,12)$.

CalTAD differs from TAD only in AP view; the apex of the femoral head is determined using a line parallel to the femoral neck that runs adjacent to the calcar rather than the center of the femoral neck (13). The maximum values of both TAD and CalTAD have been defined as $25 \mathrm{~mm}$ to prevent implant cut-out complications $(6,13)$. Many studies have shown the importance of an appropriate TAD and CaITAD to avoid implant failure $(6,7,12-15)$. In the current study, TAD and CalTAD were found to significantly differ between the two groups, with excellent inter-observer reliability. Both values were found to be higher in the cut-out group.

\section{Screw Location}

The central location of the screw is well known. The literature reports that there is a significant relationship between incorrect lag screw positioning and cut-out complication (16-18). Valentini stated that the highest 
cut-out rate was seen in the posterior section (19). De Bruijn et al. (20) reported no cut-outs in the anteriorinferior lag screws. It has also been suggested that the highest rate of cut-outs are observed in the central zone. In the current study, 27 cut-out cases were determined and when their graphs were examined, it was found that they were either superior in the AP plane or anterior in the lateral plane.

\section{Collodiaphyseal Angle}

The relationship between CDA in AP view and cut-out remains controversial $(13,21)$. In the current study, there was no significant relationship between CDA and screw cut-out.

\section{Implant Design}

The relationship between the screw cut-out complication and implant design has been evaluated in many studies, but the results are inconsistent (2224). In the current study, two types of PFN designs were used: InterTan (intertrochanteric antegrade nail) and Profin (proximal femoral intramedullary nail). InterTan PFN is made of titanium alloy and has a $4^{\circ}$ proximal valgus offset. Intertan PFN has two types with $125^{\circ}$ or $130^{\circ} \mathrm{CDA}$ and includes two screws (11 $\mathrm{mm}$ lag screw and $7 \mathrm{~mm}$ compression screw). Profin PFN is made of titanium alloy and has a $6^{\circ}$ proximal valgus offset. It is applied with $135^{\circ} \mathrm{CDA}$ and two $8.5 \mathrm{~mm}$ lag screws. The results of the current study revealed no significant relationship between the screw cut-out complication and the design of the implant used.

\section{Bone Quality and Age}

In the literature, studies report conflicting results on the relationship between bone quality and age and implant cut-out $(21,25,26)$. In the current study, no significant relationship was found between these two parameters and the cut-out complication $(\mathrm{p}=0.291$ and $\mathrm{p}=0.475$, respectively).

\section{Fracture Type}

De Brujin et al. (20) explained that AO/OTA 31-A3 fractures posed a 14 times higher cut-out risk due to their instability and difficulty of reduction. In a study evaluating 295 patients, Domingo et al. (27) reported that all the patients requiring revision procedure (3.3\%) had AO/OTA $31 \mathrm{~A} 2$ and A3 fractures. In contrast, Büyükdoğan et al. (28) suggested that fracture type was not a risk factor for the development of screw cut-out in PFN application. In the current study, $66 \%$ of the patients in the cut-out group had AO/OTA 31-A2 type fractures and 26\% had 31-A3 type fractures. There was a significant difference in fracture types between the cut-out and non-cut-out groups.

\section{Fracture Reduction}

Many studies in the literature show that there is a statistically significant relationship between nonanatomical reduction and screw cut-out $(12,20,29)$. In the current study, we observed that significantly higher rates of poor reduction in the cut-out group according to the evaluation performed using both GAI and Baumgaertner's reduction criteria.

\section{Posteromedial Cortex Continuity}

It is known that posteromedial support is an important factor for the stability of intertrochanteric fractures (30). Hao et al. (8) reported that the loss of posteromedial support was a risk factor for implant failure in AO/OTA 31-A3 fractures. In our study, we also determined that loss of posteromedial support was significantly higher in the cut-out group.

\section{Study Limitations}

This study has certain limitations. First, it was designed retrospectively. Second, the number of patients was relatively small. Third, there was no control group that included an alternative treatment method to PFN.

\section{CONCLUSION}

Fracture type, poor reduction quality, loss of posteromedial support, TAD, calcar-referenced TAD, and lag screw position were found to be associated factors in the development of screw cut-out. Apart from the type of fracture, these factors that are under the control of the surgeon generally show the importance of anatomical reduction and accurate screw placement. According to the results obtained, the risk of screw cut-out can be reduced by preoperative planning and intraoperative evaluation.

\section{ETHICAL DECLARATIONS}

Ethics Committee Approval: The study was approved by Gaziosmanpaşa Training and Research Hospital Clinical Researchs Ethics Committee (Approval date and number: 02.12.2020/192).

Informed Consent: Because the study was designed retrospectively, no written informed consent form was obtained from patients.

Referee Evaluation Process: Externally peer-reviewed.

Conflict of Interest Statement: The authors have no conflicts of interest to declare.

Financial Disclosure: The authors declared that this study has received no financial support.

Author Contributions: All of the authors declare that they have all participated in the design, execution, and analysis of the paper, and that they have approved the final version. 


\section{REFERENCES}

1. Yapici F, Ucpunar H, Camurcu Y, Emirhan N, Tanoglu O, Tardus I. Clinical and radiological outcomes of patients treated with the talon distalfix proximal femoral nail for intertrochanteric femur fractures. Injury 2020; 51: 1045-50.

2. Marsh JL, Slongo TF, Agel J, et al. Fracture and dislocation classification compendium - 2007: Orthopaedic Trauma Association classification, database and outcomes committee. J Orthop Trauma 2007; 21: S1-133.

3. Fogagnolo F, Kfuri M Jr, Paccola CA. Intramedullary fixation of pertrochanteric hip fractures with the short AOASIF proximal femoral nail. Arch Orthop Trauma Surg 2004; 124: 31-7.

4. Lenich A, Vester H, Nerlich M, Mayr E, Stöckle U, Füchtmeier B. Clinical comparison of the second and third generation of intramedullary devices for trochanteric fractures of the hip-blade vs screw. Injury 2010; 41: 1292-6.

5. Bojan AJ, Beimel C, Taglang G, Collin D, Ekholm C, Jönsson A. Critical factors in cut-out complication after Gamma Nail treatment of proximal femoral fractures. BMC Musculoskelet Disord 2013; 14: 1.

6. Zirngibl B, Biber R, Bail HJ. How to prevent cut-out and cutthrough in biaxial proximal femoral nails: is there anything beyond lag screw positioning and tip-apex distance? Int Orthop 2013; 37: 1363-8.

7. Caruso G, Andreotti M, Pari C, et al. Can TAD and CalTAD predict cut-out after extra-medullary fixation with new generation devices of proximal femoral fractures? A retrospective study. J Clin Orthop Trauma 2017; 8: 68-72.

8. Hao Y, Zhang Z, Zhou F, et al. Risk factors for implant failure in reverse oblique and transverse intertrochanteric fractures treated with proximal femoral nail antirotation (PFNA). J Orthop Surg Res 2019; 14: 350.

9. Li C, Xie B, Chen S, Lin G, Yang G, Zhang L. The effect of local bone density on mechanical failure after internal fixation of pertrochanteric fractures. Arch Orthop Trauma Surg 2016; 136: 223-32.

10. Caruso G, Bonomo M, Valpiani G, et al. A six-year retrospective analysis of cut-out risk predictors in cephalomedullary nailing for pertrochanteric fractures: Can the tip-apex distance (TAD) still be considered the best parameter? Bone Joint Res 2017; 6: 481-8.

11.Landis JR, Koch GG. The measurement of observer agreement for categorical data. Biometrics 1977; 33: 159-74.

12.Koyuncu Ş, Altay T, Kayalı C, Ozan F, Yamak K. Mechanical failures after fixation with proximal femoral nail and risk factors. Clin Interv Aging 2015; 10: 1959-65.

13. Kashigar A, Vincent A, Gunton MJ, Backstein D, Safir O, Kuzyk PR. Predictors of failure for cephalomedullary nailing of proximal femoral fractures. Bone Joint J 2014; 96: 1029-34.

14. Hsueh KK, Fang CK, Chen CM, Su YP, Wu HF, Chiu FY. Risk factors in cutout of sliding hip screw in intertrochanteric fractures: an evaluation of 937 patients. Int Orthop 2010; 34: 1273-6.

15. Geller JA, Saifi C, Morrison TA, Macaulay W. Tip-apex distance of intramedullary devices as a predictor of cut-out failure in the treatment of peritrochanteric elderly hip fractures. Int Orthop 2010; 34: 719-22.

16. Schipper IB, Steyerberg EW, Castelein RM, et al. Treatment of unstable trochanteric fractures. Randomised comparison of the gamma nail and the proximal femoral nail. J Bone Joint Surg Br 2004; 86: 86-94.

17. Menezes DF, Gamulin A, Noesberger B. Is the proximal femoral nail a suitable implant for treatment of all trochanteric fractures? Clin Orthop Relat Res 2005; 439: 221-7.

18. Uzun M, Ertürer E, Oztürk I, Akman S, Seçkin F, Ozçelik IB. Longterm radiographic complications following treatment of unstable intertrochanteric femoral fractures with the proximal femoral nail and effects on functional results. Acta Orthop Traumatol Turc 2009; 43: 457-63.
19. Valentini R, Martino M, Piovan G, De Fabrizio G, Fancellu G. Proximal cut-out in pertrochanteric femural fracture. Acta Biomed 2014; 85: 144-51.

20.De Bruijn K, den Hartog D, Tuinebreijer W, Roukema G. Reliability of predictors for screw cutout in intertrochanteric hip fractures. J Bone Joint Surg Am 2012; 94: 1266-72.

21.Lobo-Escolar A, Joven E, Iglesias D, Herrera A. Predictive factors for cutting-outin femoral intramedullary nailing. Injury 2010; 41 : 1312-6.

22. Audige L, Hanson B, Swiontkowski MF. Implant-related complications in the treatment of unstable intertrochanteric fractures: meta-analysis of dynamic screw-plate versus dynamic screw-intramedullary nail devices. Int Orthop 2003, 27: 197-203.

23. Tsai SW, Lin CJ, Tzeng YH, et al. Risk factors for cut-out failure of Gamma3 nails in treating unstable intertrochanteric fractures: An analysis of 176 patients. J Chin Med Assoc 2017; 80: 587-94.

24.Duramaz A, İlter $\mathrm{MH}$. The impact of proximal femoral nail type on clinical and radiological outcomes in the treatment of intertrochanteric femur fractures: a comparative study. Eur J Orthop Surg Traumatol 2019; 29: 1441-9.

25.Klatte TO, Vettorazzi E, Beckmann J, Pueschel K, Amling M, Gebauer M. The Singh Index does not correlate with bone mineral density (BMD) measured withdual energy X-ray absorptiometry (DXA) or peripheral quantitative computedtomography (pQCT). Arch Orthop Trauma Surg 2015; 135: 645-50.

26. Morvan A, Boddaert J, Cohen-Bittan J, Picard H, PascalMousselard H, Khiami F. Risk factors for cut-out after internal fixation of trochanteric fractures in elderly subjects. Orthop Traumatol Surg Res 2018; 104: 1183-7.

27. Domingo LJ, Cecilia D, Herrera A, Resines C. Trochanteric fractures treated with a proximal femoral nail. Int Orthop 2001; 25: 298-301.

28. Buyukdogan K, Caglar O, Isik S, Tokgozoglu M, Atilla B. Risk factors for cut-out of double lag screw fixation in proximal femoral fractures. Injury 2017; 48: 414-8.

29. Basılgan S, Büyükkuşcu MÖ. Treatment criteria and methods in osteosynthesis failure. TOTBİD Derg 2021; 20: 1-7.

30. Marmor M, Liddle K, Pekmezci M, Buckley J, Matityahu A. The effect of fracture pattern stability on implant loading in OTA type 31-A2 proximal femur fractures. J Orthop Trauma 2013; 27: 683-9. 